\title{
Determination of Constitutive Parameters of Plastic Materials Using a Finite-Element Polycrystalline Model*
}

\author{
Moriaki GOYA** and Koichi ITO***
}

\begin{abstract}
We previously proposed a constitutive expression of plastic deformation which can incorporate the directional dependence of the plastic strain increment $\dot{\boldsymbol{\varepsilon}}^{P}$ on the stress increment $\dot{\sigma}$. The expression was given in terms of two transition parameters $\mu(\alpha)$ and $\beta(\alpha)$ which denote the magnitude and the direction angle of the plastic increment, where $\alpha$ denotes the direction angle of the stress increment measured from a particular direction, termed "natural direction", in which the direction of the stress increment coincides with that of the plastic strain increment.

The expression can be utilized once we obtain the parameters $\mu(\alpha)$ and $\beta(\alpha)$ through experimentation on industrial materials and/or theoretical studies such as polycrystalline model analyses. In this report, a computer code for a finite-element polycrystalline model is developed and used for the investigation of the variations of the two constitutive parameters $\mu(\alpha)$ and $\beta(\alpha)$.
\end{abstract}

Key Words: Plasticity, Constitutive Relation, Finite-Element Method, Polycrystalline Model, Numerical Analysis, Crystal Plasticity, Directional Dependence Rule

\section{Introduction}

At critical loading points in most bifurcation problems such as the buckling of shells and localization of plates, a new deformation mode or a new strain field abruptly appears, which is quite different from a preceding fundamental deformation mode. For example, Hill(1) dealt with the localized deformation as a bifurcating mode from a uniform deformation of a plastic plate subjected to in-plane loading. It is well known that Hill's criterion for the localization of the plastic plate does not result in a limit curve in the loading region where both principal strains, $\varepsilon_{1}$ and $\varepsilon_{2}$, are simultaneously positive, although the localization of plastic plates was observed in the loading region in numerous experimental studies in the past. The discrepancy between the predictions by Hill's criterion and past experimental results has been found to be due to the fact that Hill's criterion is based on the $J_{2}$-flow rule, namely the Prandtl-Reuss flow rule,

* Received 7th November, 1996.

** Department of Mechanical Systems Engineering, Univ. of the Ryukyus, 1 Sembaru, Nishihara-cho, Okinawa 903-01, Japan

*** Department of Computer and Mathematical Science, Tohoku University, Aoba, Sendai, Miyagi 980, Japan which does not permit the plastic strain increment to change direction according to the direction of the stress increment. Therefore, the application of the $J_{2^{-}}$ flow rule to such a bifurcation problem necessarily means confining the possible deformation of the bifurcation to within certain deformation modes.

In 1975, to eliminate the defect of Hill's criterion, Stören and Rice ${ }^{(2)}$ introduced to their bifurcation analysis an incremental form of Hencky's deformation rule, which assures a one-to-one relationship between the increments of stress and plastic strain and permits directional dependence; the equation permits the direction of the plastic strain increment to change according to the direction of the stress increment. The original nonincremental form of Hencky's deformation rule is not accepted as a rigorous one since the original form is ambiguous with respect to unloading and reloading conditions which are indispensable in plasticity. Batdorf and Budiansky, however, clarified ${ }^{(3)}$ that the incremental form of Hencky's deformation rule can be reasonable if we introduce a corner on the yield surface at the current loading point. Motivated by this research, some phenomenological corner theories have been proposed by Christoffersen \& Hutchinson ${ }^{(4)}$ and by Gotoh $^{(5)}$. Then, Gotoh ${ }^{(6)}$ proposed a nonassociate flow rule in 
1989, which permits directional dependence without corner formation.

The authors ${ }^{(7)}$ independently proposed a plastic constitutive expression that can incorporate the directional dependence of the plastic strain increment. The equation is expressed with the use of two transition parameters $\mu(\alpha)$ and $\beta(\alpha)$ which denote the magnitude and the direction angle of the plastic increment; $\alpha$ denotes the direction angle of the stress increment measured from a particular direction termed "natural direction" in which the direction of the stress increment coincides with that of the plastic strain increment. It has been demonstrated that the introduced parameters are essential for describing incremental constitutive properties of plastic materials and that the proposed equation is different from the ones developed by Christoffersen \& Hutchinson ${ }^{(4)}$ and by Gotoh $^{(5),(6)}$.

However, Goya and Ito's equation ${ }^{(7)}$ can be reduced to the corner rules or the nonassociate flow rule developed by Christoffersen \& Hutchinson and by Gotoh simply by choosing the introduced parameters appropriately. This generality of Goya and Ito's equation is due to the introduction of two transition parameters $\mu(\alpha)$ and $\beta(\alpha)$.

Direct experimental determination of the constitutive properties of plasticity requires precise measurements and a number of specimens with exactly the same dimensions and material properties to reach a reliable conclusion. In fact, even for the confirmation of corner formation, some researchers experimental data support the formation but others do not. It should, therefore, be noted that experimental determination of the precise forms of the parameters is a very difficult task since we must prepare a large number of specimens of the same initial material properties, subject them to loading to achieve a certain level of stress in a specified stress direction to establish an identical loading history, and then carefully but abruptly change the loading direction in various orientations to obtain $\mu(\alpha)$ and $\beta(\alpha)$ for one stress direction.

Therefore, numerical simulations based on crystal plasticity have been regarded as a useful tool for experimental determination of $\mu(\alpha)$ and $\beta(\alpha)$. In 1979, to study the directional dependence of the plastic strain increment Ito et al..$^{(8)}$ used the KBW self-consistent model, which was proposed by Kroner ${ }^{(9)}$ and Budiansky \& $W_{u^{(10)}}$. Then, in 1990 , to study the same problem Takahashi et al. ${ }^{(1)}$ used Lin's polycrystal model in which a three-dimensional solid is assumed to be composed of numerous identical cubic blocks. The results from both studies clearly show the directional dependence of the plastic strain increment although the degree of the dependence may change depending on the loading direction and the current stress state. The applicability of the theories proposed in both studies, however, is restricted to certain stress states since the theories are based on small deformation and cannot take into account neither large deformation nor crystal rotation.

In recent years, finite-element polycrystalline models, denoted as FEPM hereafter, have been developed based on the large deformation theory, and can be applied to various problems of plasticity. For example, in 1994, Takahashi et al. ${ }^{(12)}$ developed a FEPM to study the evolution of the anisotropic aspects of plastic materials induced by the lattice rotation due to large plastic deformation. In this report, we develop a FEPM to study the variations of the transition parameters $\mu(\alpha)$ and $\beta(\alpha)$.

\section{Constitutive Equation Incorporating Stress Increment Directional Dependence (Goya \& Ito $^{(7)}$ )}

The following constitutive equation was proposed by Goya and Ito for elastic-plastic materials : If $N n$ :

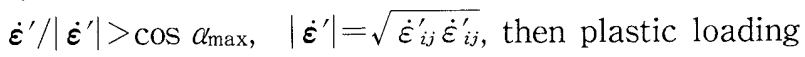
occurs and Eq. ( 1 ) holds.

$$
\dot{\boldsymbol{\sigma}}=2 G^{*}\left\{\dot{\boldsymbol{\varepsilon}}^{\prime}+\nu^{*} /(1-2 \nu) \dot{\boldsymbol{\varepsilon}}^{V} \boldsymbol{\delta}-\left(\boldsymbol{N} n: \dot{\boldsymbol{\varepsilon}}^{\prime}\right) \boldsymbol{N} n / S^{*}\right\},
$$

where the symbols used in Eq. (1) are defined as follows:

$\begin{aligned} \dot{\varepsilon}^{V} & : \text { Volumetric strain increment } \\ \nu & : \text { Poisson's ratio } \\ G^{*} & =G H^{\prime} /\left(H^{\prime}+3 G \mu \sin \beta / \sin \alpha\right) \\ S^{*} & =\left\{H^{\prime} \sin 2 \alpha /(6 \mu G)+\sin \alpha \cos \beta\right\} / \sin (\alpha-\beta)\end{aligned}$

$G$ : Elastic shear modulus

$H^{\prime}$ : Tangential coefficient of stress strain curve

$\nu^{*}=\nu+E \mu \sin \beta /\left(2 H^{\prime} \sin \alpha\right)$

$\boldsymbol{\delta}$ : Kronecker's delta tensor

()$^{\prime}$ : Deviatoric component of () , and $\alpha$ and $\beta$ are direction angles of the stress increment and of the plastic strain increment, respectively, which are measured from the natural direction vector $N n$. Figure 1 shows the relation among some fundamental quantities.

For Mises-type isotropic materials, the natural direction coincides with that of the deviatoric stress vector itself. For non-Mises materials such as Tresca materials which are isotropic, or for anisotropic materials, it may hold that the natural direction coincides with that of the outward normal at the current stress point on the yield surface. The directional dependence means specifically that the plastic strain increment direction angle, $\beta$, is a function of $\alpha$. 


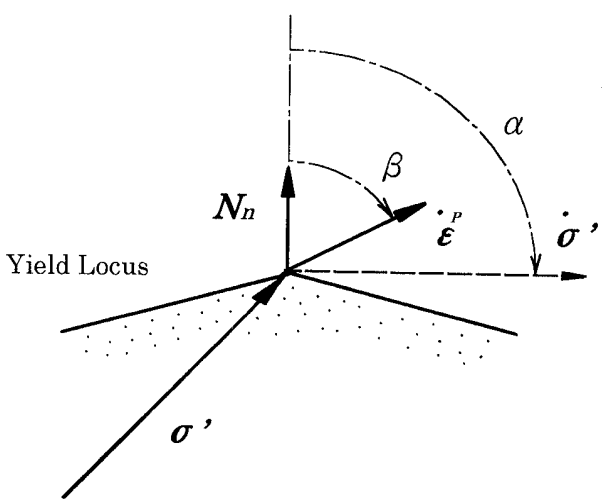

Fig. 1 Geometrical relationships among yield locus in the stress space, angular measures, stress increment, natural direction vector and plastic strain increment at a plastic loading point

The magnitude of the plastic strain increment also depends upon $\alpha$, and the following relation holds between the increments of stress and plastic strain using the magnitude parameter, $\mu(\alpha)$, of the plastic strain increment

$$
\overline{\dot{\sigma}}=H^{\prime} \overline{\dot{\varepsilon}^{P}} / \mu(\alpha)
$$

Here, $H^{\prime}$ is the work-hardening coefficient for the incremental loading in the natural direction, $\overline{\dot{\sigma}}=$

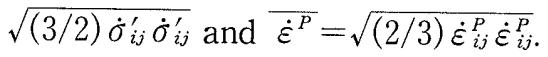

It should be noted that $\mu(\alpha)$ is reduced to $\cos (\alpha)$ and $\beta$ to 0 for the $J_{2}-$ flow rule. It has been proved that Eq. (1) ensures one-to-one correspondence between strain increments $\dot{\varepsilon}$ and stress increments $\dot{\sigma}$, when the transition parameters satisfy the following conditions within the range $0 \leq \alpha \leq \alpha_{\max }$ :

( i ) $\mu(0)=1$ and $\mu\left(\alpha_{\max }\right)=0$, and

$\mu(\alpha)$ decreases monotonically with respect to $\alpha$.

(ii) $\beta(0)=0$ and $\beta\left(\alpha_{\max }\right)=\beta_{\max }$, and

$\beta(\alpha)$ increases monotonically with respect to $\alpha$.

\section{Finite-Element Polycrystalline Model(FEPM)}

The FEPM is a numerical method in which each element or Gaussian integration point is regarded as a single crystal. Then, mechanical properties of a single crystal are allocated to the elements or Gaussian points. As a result of the allocation, a block composed of the elements can be regarded as a polycrystalline aggregate to numerically obtain the macroscopic response of a plastic material. In 1971, Havner ${ }^{(13)}$ proposed a finite-element model to investigate the effect of microscopic crystal plasticity mechanism on the macroscopic response as a polycrystalline aggregate. In 1972, Jimma $^{(14)}$ and Miyamoto ${ }^{(15)}$ independently proposed a procedure to analyze the behavior of activated slip systems. Their studies, however, were performed under the assumption of the small deformation. In 1978, Gotoh ${ }^{(16)}$ reported on a FEPM taking into account the rotation of crystal orientation due to plastic deformation. In 1979, Asaro $^{(17)}$ developed a planar double slipping crystal model based on the theory proposed by Asaro \& Rice ${ }^{(18)}$. Takahashi et al. ${ }^{(12)}$ in 1994 developed a computer code for FEPM for non-work-hardening materials based on an incremental, initial strain scheme taking into account a large deformation effect. In this section, we discuss a procedure for introducing a constitutive relation expressed in the crystallographic coordinate system, termed the local coordinate system hereafter, according to the works of Asaro \& Rice ${ }^{(18)}$ and Gotoh ${ }^{(16)}$, and then develop a computer code for FEPM based on a three-dimensional formulation to study the precise forms of the constitutive transition parameters $\mu(\alpha)$ and $\beta(\alpha)$ of face centered cubic (FCC) materials considering work-hardening and such large deformation effects as geometrical nonlinearity and the lattice rotation.

\section{1 Crystal Plasticity}

We assume that the plastic deformation of a single crystal is caused only by crystallographic slips along slip systems of finite number $n$; the effects of twinning formation and slips at grain boundaries will be neglected. Furthermore, we assume that elastic properties are not affected by the slips, that the total strain increment $\dot{\boldsymbol{\varepsilon}}$ can be decomposed into $\dot{\boldsymbol{\varepsilon}}^{e}$ and $\dot{\boldsymbol{\varepsilon}}^{P}$ and that the corotational Kirchhoff stress increment $\dot{\sigma}^{c}$, corotational with the lattice of the crystal and defined as $\dot{\boldsymbol{\sigma}}^{c}=\dot{\boldsymbol{\sigma}}-\boldsymbol{\Omega} \boldsymbol{\sigma}+\boldsymbol{\Omega} \boldsymbol{\sigma}$ using the lattice spin $\boldsymbol{\Omega}$, is related to $\dot{\varepsilon}^{e}$ as

$$
\left\{\dot{\sigma}^{C}\right\}=\left[C^{e}\right]\left\{\dot{\varepsilon}^{e}\right\}=\left[C^{e}\right]\{\dot{\varepsilon}\}-\left[C^{e}\right]\left\{\dot{\varepsilon}^{P}\right\},
$$

where $\{\dot{\sigma}\}$ denotes the stress increment vector evaluated for the local coordinate system of each single crystal, $\{\dot{\varepsilon}\}$ the corresponding strain increment vector and $\left[C^{e}\right]$ is the elastic constitutive matrix of the single crystal described according to the local coordinate system.

As regards to deformation of a single crystal, the shear strain increment $\dot{\gamma}^{P}$ of slip systems is related to the corresponding plastic strain increment $\dot{\varepsilon}^{P}$ as follows in the tensorial expression:

$$
\dot{\boldsymbol{\varepsilon}}^{p}=(1 / 2) \Sigma\left(\boldsymbol{p}_{k} \otimes \boldsymbol{q}_{k}+\boldsymbol{q}_{k} \otimes \boldsymbol{p}_{k}\right) \dot{\gamma}_{k}^{p},
$$

where $\boldsymbol{p}_{k}$ is a unit vector along the $k$-th slip system and $\boldsymbol{q}_{k}$ is a unit vector orthogonal to the slip system.

Equation ( 7 ) can be rewritten as follows for FCC materials :

$$
\left\{\dot{\varepsilon}^{P}\right\}=[T]^{T}\left\{\dot{\gamma}^{P}\right\}
$$

The shear stress, $\tau_{k}$, on the $k$-th slip system is obtained from

$$
\tau_{k}=\boldsymbol{p}_{k} \cdot \boldsymbol{\sigma} \boldsymbol{q}_{k}
$$

Equation (9) is the conjugate relation of Eq. ( 7 ) in view of making the energy rate such that $\sum \tau_{k} \dot{\gamma}_{k}^{P}$ and $\sigma: \dot{\boldsymbol{\varepsilon}}^{P}$. From Eq. (9) the following holds between 
Table 1 Details of matrix $\left[T_{S}\right]$

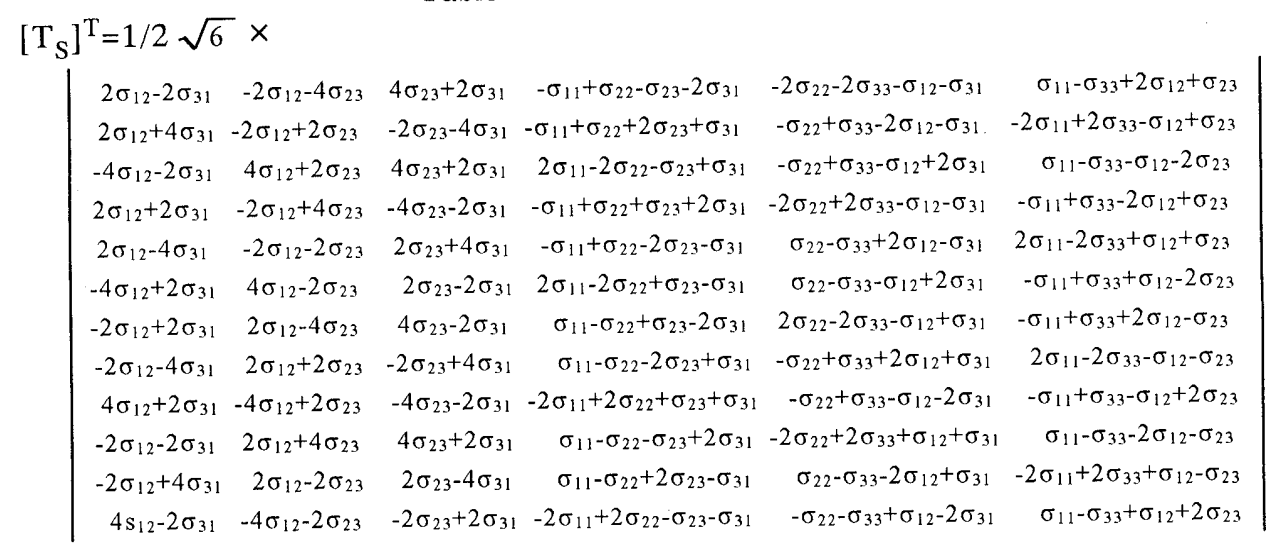

shear stress $\{\tau\}$ on slip systems and Cauchy stress $\{\sigma\}$ as

$$
\{\tau\}=[T]\{\sigma\}
$$

Asaro and Rice ${ }^{(18)}$ discussed the details of the incremental form of Eq. ( 9 ) to study the appropriate definition of the corotational rate of $\boldsymbol{\sigma}, \boldsymbol{q}_{k}$ and $\boldsymbol{p}_{k}$. According to their results, if we choose $\boldsymbol{q}_{k}$ and $\boldsymbol{p}_{k}$ to be orthogonal unit vectors during deformation but simply rotate them rigidly by the lattice spin rate, $\boldsymbol{\Omega}$, the following holds :

$$
\dot{\boldsymbol{p}}_{k}=\boldsymbol{\Omega} \boldsymbol{p}_{k}, \quad \dot{\boldsymbol{q}}_{k}=\boldsymbol{\Omega} \boldsymbol{q}_{k},
$$

where $\boldsymbol{W}=\boldsymbol{\Omega}+\boldsymbol{\omega}^{s}, \boldsymbol{W}$ is the conventional continuum spin tensor and $\omega^{S}$ is related to shear strain $\dot{\boldsymbol{\gamma}}^{P}$ of active slip systems as

$$
\boldsymbol{\omega}^{S}=(1 / 2) \sum\left(\boldsymbol{p}_{k} \otimes \boldsymbol{q}_{k}-\boldsymbol{q}_{k} \otimes \boldsymbol{p}_{k}\right) \dot{\gamma}_{k}^{P} .
$$

Here, it should be noted that $\boldsymbol{\Omega}$ is due to the lattice spin.

Then, the incremental resolved shear stress $\dot{\tau}_{k}$, occurring in a single crystal, is given by the corotational stress increment $\dot{\sigma}^{c}$ as

$$
\dot{\tau}_{k}=\boldsymbol{p}_{k} \cdot \dot{\boldsymbol{\sigma}}^{C} \boldsymbol{q}_{k} \text {, }
$$

which can be written as $\{\dot{\tau}\}=[T]\left\{\dot{\sigma}^{c}\right\}$, where $\left\{\dot{\sigma}^{c}\right\}$ is defined as the Kirchhoff stress increment taking into account the lattice spin.

Substituting Eq. ( 8$)$ into Eq. (6), we get

$$
\left\{\dot{\sigma}^{c}\right\}=\left[C^{e}\right]\left\{\dot{\varepsilon}^{e}\right\}-\left[C^{e}\right][T]^{T}\left\{\dot{\gamma}^{P}\right\} \text {. }
$$

The Jaumann increment of Kirchhoff stress $\left\{\dot{\sigma}^{J}\right\}$ evaluated, taking into account the conventional continuum spin, is related to the increment $\left\{\dot{\sigma}^{c}\right\}$ that is corotational with the lattice spin $\operatorname{as}^{(16)}$

$$
\left\{\dot{\sigma}^{J}\right\}=\left\{\dot{\sigma}^{c}\right\}+\left[\omega^{s}\right]\{\sigma\},
$$

where $\left[\omega^{s}\right]$ is the spin-effect matrix composed of corotational spin due to crystallographic slips given by Eq. (12), and is defined as

$$
\left[\omega^{S}\right]=\left[\begin{array}{cccccc}
0 & 0 & 0 & 2 \omega_{21}^{S} & 0 & 2 \omega_{31}^{S} \\
0 & 0 & 0 & 2 \omega_{12}^{S} & 2 \omega_{32}^{S} & 0 \\
0 & 0 & 0 & 0 & 2 \omega_{23}^{S} & 2 \omega_{13}^{S} \\
\omega_{12}^{S} & \omega_{21}^{S} & 0 & 0 & \omega_{31}^{S} & \omega_{32}^{S} \\
0 & \omega_{23}^{S} & \omega_{32}^{S} & \omega_{13}^{S} & 0 & \omega_{12}^{S} \\
\omega_{13}^{S} & 0 & \omega_{31}^{S} & \omega_{23}^{S} & \omega_{21}^{S} & 0
\end{array}\right] .
$$

For slip systems of a single crystal, we assume the following constitutive relationship between the plastic shear strain increment vector and the shear stress increment vector when the slip systems are activated:

$$
\{\dot{\tau}\}=\left[h_{a b} \operatorname{sign}\left(\tau_{b}\right)\right]\left\{\dot{\gamma}^{P}\right\}
$$

where $h_{a b}$ is the instantaneous hardening coefficient of slip planes and is assumed to be given by $h_{a b}=H^{\prime}\{q$ $\left.+(1-q) \delta_{a b}\right\}^{(17)}$ and $q$ denotes the ratio of latent-hardening to self-hardening. Usually $q=1 \sim 1$.4. When $q$ $=1$, Eq. (17) is identical to Taylor's hardening law, namely the isotropic hardening law, and $q=0$ reduces Eq. (17) to non latent hardening law.

Using Eqs. (13) to (17), we obtain

$$
\left[h_{a b} \operatorname{sign}\left(\tau_{b}\right)\right]\left\{\dot{\gamma}^{P}\right\}=[T]\left(\left[C^{e}\right]\{\dot{\varepsilon}\}\right.
$$

$$
\left.-\left[C^{e}\right][T]^{T}\left\{\dot{\gamma}^{P}\right\}-\left[\omega^{S}\right]\{\sigma\}\right) \text {. }
$$

Then, introducing $\left[T_{S}\right]$ defined by $\left[\omega^{S}\right]\{\sigma\}=\left[T_{S}\right]\left\{\dot{\gamma}^{P}\right\}$, we can rewrite Eq. (18) as

$$
\begin{gathered}
{\left[\Sigma h_{a b} \operatorname{sign}\left(\tau_{b}\right)\right]\left\{\dot{\gamma}^{P}\right\}+\left([T]\left[C^{e}\right][T]^{T}\right.} \\
\left.+[T]\left[T_{s}\right]\right)\left\{\dot{\gamma}^{P}\right\}=[T]\left[C^{e}\right]\{\dot{\varepsilon}\},
\end{gathered}
$$

where the details of $\left[T_{S}\right]$ are given in Table 1 .

We can solve Eq. (19) for $\left\{\dot{\gamma}^{P}\right\}$ as

$\left\{\dot{\gamma}^{P}\right\}=[\Gamma]\{\dot{\varepsilon}\}$,

where $[\Gamma]=[P]^{-1}[T]\left[C^{e}\right]$ and

$$
\left.[P]=\left[h_{a b} \operatorname{sign}\left(\tau_{b}\right)\right]+[T]\left[C^{e}\right][T]^{T}+[T]\left[T_{s}\right]\right) .
$$

From Eqs. (14), (15) and (20), the following equation is derived as a constitutive relation expressed in the local coordinate system

$$
\left\{\dot{\sigma}^{J}\right\}=\left[C^{e}\right]\left[[I]-\left([T]^{T}-\left[C^{e}\right]^{-1}\left[T_{s}\right]\right)[\Gamma]\right]\{\dot{\varepsilon}\} .
$$

Equation (22) can be converted into the constitutive relation expressed in the global coordinate system

$$
\left\{\dot{S}^{J}\right\}=[\Phi]^{T}\left\{\dot{\sigma}^{J}\right\}=[D]\{\dot{E}\}
$$

where,

$$
[D]=[\Phi]^{T}\left[C^{e}\right]\left[[I]-\left([T]^{T}-\left[C^{e}\right]^{-1}\left[T_{S}\right]\right)[\Gamma]\right][\Phi],
$$


Table 2 Relationships between direction cosines $(l, m, n)$ and Euler angels

\begin{tabular}{|c|ccc|}
\hline & $x_{1}$ & $x_{2}$ & $x_{3}$ \\
\hline $\mathrm{x}_{1}$ & $l_{1}(=\cos \phi \cos \theta \cos \psi-\sin \theta \sin \psi)$ & $l_{2}(=-\sin \psi \cos \phi \cos \theta-\sin \theta \cos \psi)$ & $l_{3}(=\cos \theta \sin \phi)$ \\
$\mathrm{x}_{2}$ & $m_{1}(=\cos \phi \sin \theta \cos \psi+\cos \theta \sin \psi)$ & $m_{2}(=-\sin \psi \cos \phi \sin \theta+\cos \theta \cos \psi)$ & $m_{3}(=\sin \theta \sin \phi)$ \\
$\mathrm{x}_{3}$ & $n_{1}(=-\sin \phi \cos \psi)$ & $n_{2}(=\sin \phi \sin \psi)$ & $n_{3}(=\cos \phi)$ \\
\hline
\end{tabular}

$\mathbf{X}$; Global coordinate system, Xi; Local coordinate system

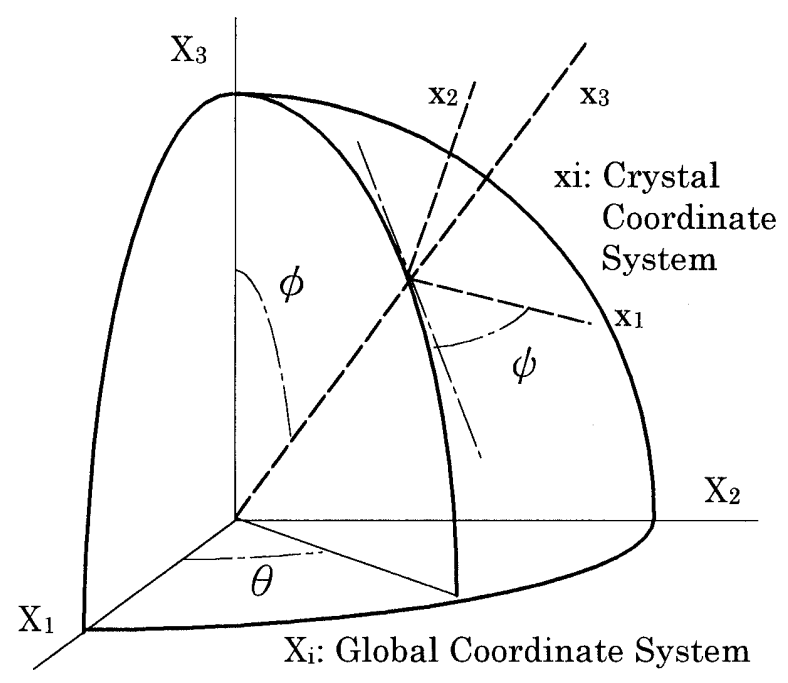

Fig. 2 Global coordinate system $\left(X_{1}, X_{2}, X_{3}\right)$ fixed in the space, local (or crystal) coordinate $\operatorname{system}\left(x_{1}, x_{2}\right.$, $\left.x_{3}\right)$ and Euler angles defining the orientation difference between the local coordinates and global coordinates

$$
\Phi \Phi]=\left[\begin{array}{cccc}
l_{1}^{2} & m_{1}^{2} & n_{1}^{2} & l_{1} m_{1} \\
l_{2}^{2} & m_{2}^{2} & n_{2}^{2} & l_{2} m_{2} \\
l_{3}^{2} & m_{3}^{2} & n_{3}^{2} & l_{3} m_{3} \\
2 l_{1} l_{2} & 2 m_{1} m_{2} & 2 n_{1} n_{2} & l_{1} m_{2}+l_{2} m_{1} \\
2 l_{2} l_{3} & 2 m_{2} m_{3} & 2 n_{2} n_{3} & l_{2} m_{3}+l_{3} m_{2} \\
2 l_{3} l_{1} & 2 m_{3} m_{1} & 2 n_{3} n_{1} & l_{3} m_{1}+l_{1} m_{3} \\
m_{1} n_{1} & n_{1} l_{1} \\
m_{2} n_{2} & n_{2} l_{2} \\
m_{3} n_{3} & n_{3} l_{3} \\
m_{1} n_{2}+m_{2} n_{1} & n_{1} l_{2}+n_{2} l_{1} \\
m_{2} n_{3}+m_{3} n_{2} & n_{2} l_{3}+n_{3} l_{2} \\
m_{3} n_{1}+m_{1} n_{3} & n_{3} l_{1}+n_{1} l_{3}
\end{array}\right]
$$

and $l_{k}, m_{k}$ and $n_{k}(k=1,2,3)$ are the direction cosines defining the orientation of the local coordinate system with respect to the global coordinate system. The direction cosines are given in Table 2 using Euler's angles that relate the axis directions of the local coordinate system to those of the global coordinate system as shown in Fig. 2.

The constitutive matrix defined by Eq. (24) is used for constructing a stiffness matrix in the finite-element code. It should be noted that the aforementioned derivation of Eq. (24) is based on the assumption that we can identify the actually activated slips in each time increment. This problem of the identification of active slip systems is a crucial issue of rate-independent polycrystalline plasticity and has been discussed by many researchers, such as Havner ${ }^{(13)}$, Takahashi et al. ${ }^{(12)}$, Asaro ${ }^{(17)}$ and Balasubramanian ${ }^{(19)}$. These researchers proposed their own scheme to solve or overcome this issue. In this study, we use the $r_{\mathrm{min}}{ }^{-}$ scheme, proposed by Yamada ${ }^{(20)}$ as an explicit solution for plasticity problems, to control the magnitude of deformation within one incremental time step. Slip systems are regarded as activated at the next time step, if $\left|\tau_{k}\right|=Y_{k}$ (yield stress of the $k$-th slip system) and $\tau_{k} * \dot{\tau}_{k}>0$ but regarded as nonactivated when $\left|\tau_{k}\right|$ $=Y_{k}$ and $\tau_{k} * \dot{\tau}_{k}<0$, in which case a very small value is allocated to $r_{\min }$.

\section{2 Lattice rotation}

For large deformation problems of crystalline materials, the evolution equation of the Euler angles must be obtained because the rotation of the crystal lattice cannot be ignored. Here, we start our discussion based on the following relation among the spins expressed in the global coordinate system

$$
[W]=[\Omega]+\left[W^{s}\right]
$$

where $[W]$ is the conventional continuum spin, $[\Omega]$ is the spin due to the lattice deformation and rotation and $\left[W^{s}\right]$ is a pseudospin in view of lattice rotation since we assume that the lattice orientation is not affected by the sliding of slip systems.

Then, the net lattice spin $[\Omega]$ is related to the orthogonal matrix $[R]$, the coordinate transformation matrix shown in Table 2 , as

$$
[\dot{R}]=[R][\Omega]
$$

where it should be noted that $[\Omega]$ is expressed in the global coordinate system.

From a comparison of corresponding terms on the right hand side of Eq. (27) and the time derivative of $[R]$, we obtain

$$
\{\dot{\Phi}\}=\left[R_{\text {rot }}\right]\{\Omega\},
$$

where $\{\dot{\Phi}\}^{T}=\{\dot{\phi}, \dot{\phi}, \dot{\theta}\}, \quad\{\Omega\}^{T}=\left\{\Omega_{12}, \Omega_{23}, \Omega_{31}\right\}$ and

$$
\left[R_{\text {rot }}\right]=\left[\begin{array}{ccc}
0 & -\sin \phi & -\cos \phi \\
0 & \cos \phi / \sin \phi & -\sin \phi / \sin \phi \\
-1 & -\cot \phi \cos \phi & \cot \phi \sin \phi
\end{array}\right] \text {. }
$$

We also note that the following holds between $\left\{W^{S}\right\}$, the lattice spin vector expressed in the global 
coordinate system, and $\left\{\omega^{S}\right\}$, the lattice spin vector expressed in the local coordinate system as shown in Eq. (12)

$$
\left\{W^{s}\right\}=\left[T_{\text {trns }}\right]\left\{\omega^{S}\right\},
$$

where $\quad\left\{W^{S}\right\}^{T}=\left\{W_{12}^{S}, W_{23}^{S}, W_{31}^{S}\right\}, \quad\left\{\omega^{S}\right\}^{T}=\left\{\omega_{12}^{S}, \omega_{23}^{S}, \omega_{31}^{S}\right\}$ and

$$
\left[T_{\text {trns }}\right]=\left[\begin{array}{ccc}
l_{1} m_{2}-l_{2} m_{1} & l_{2} m_{3}-l_{3} m_{2} & l_{3} m_{1}-l_{1} m_{3} \\
m_{1} n_{2}-m_{2} n_{1} & m_{2} n_{3}-m_{3} n_{2} & m_{3} n_{1}-m_{1} n_{3} \\
n_{1} l_{2}-n_{2} l_{1} & n_{2} l_{3}-n_{3} l_{2} & n_{3} l_{1}-n_{1} l_{3}
\end{array}\right] .
$$

Furthermore, from Eq. (12), we obtain

$$
\left\{\omega^{S}\right\}=\left[T_{\text {rot }}\right]\left\{\dot{\gamma}^{P}\right\} .
$$

Now, we can obtain the evolution equation of the Euler angles from Eqs. (26), (28), (30) and (32) as

$$
\{\dot{\Phi}\}=[R]\left[\{W\}-\left[T_{\text {trns }}\right]\left[T_{\text {rot }}\right]\left\{\dot{\gamma}^{P}\right\}\right] .
$$

\section{3 Finite-element formulation}

The finite-element code for the analysis of polycrystalline materials is based on the tangential coefficient scheme and the following virtual work principle

$$
\begin{gathered}
\int_{V}\left\{\left(\dot{S}_{i j}^{J}-2 S_{i j} \dot{E}_{k j}\right) \delta \dot{E}_{i j}+\sum_{j k} L_{i k} \delta L_{i j}\right\} d V \\
=\int_{S} \dot{t}_{i} \delta v_{i} d S+\int_{V} \dot{b}_{i} \delta v_{i} d V
\end{gathered}
$$

where the incremental constitutive law is given by Eq. (23) as a relation between the Jaumann increment of the Kirchhoff stress $\dot{S}_{i j}^{I}$ and the stretching tensor $\dot{E}_{i j}$.

\section{Numerical Model and Calculation Conditions}

The polycrystalline model block consisting of 64 eight-node-isoparametric elements is introduced as shown in Fig. 3. Each element is regarded as a single crystal ; for each element, the same crystal orientations are given at eight Gaussian integration points at the initial state. The Euler angles of the crystal coordinate systems are chosen by a random number generator. We choose the material constants as Young's modulus $E=72 \mathrm{GPa}$, Poisson's ratio $\nu=0.3$, initial shear yield stress of slip systems $Y=200 \mathrm{MPa}$,

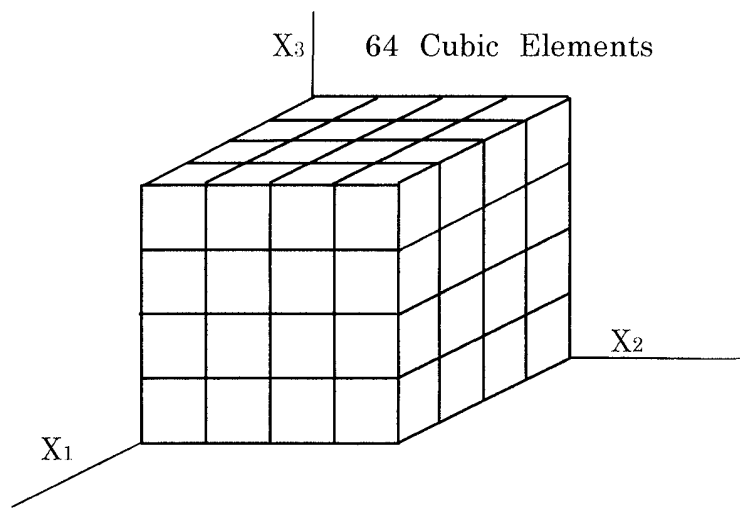

Fig. 3 Finite-element divisions of a polycrystalline model block introduced in the study work-hardening coefficient $H^{\prime}=800 \mathrm{MPa}$ and latent work-hardening parameter $q=1.2$. We regard the model block in Fig. 3 as one-eighth of the entire region because of the symmetry of the problem and the boundary conditions are given for the block such that the entire region is deformed by uniformly distributing symmetric nodal forces. After deforming the model along a proportional loading direction up to a certain level of strain, we set nodal forces to different values to change the macroscopic stress direction from the proportional direction. This process gives values of $\mu\left(\alpha_{A}\right)$ and $\beta\left(\alpha_{A}\right)$ for a direction angle $\alpha_{A}$.

The macroscopic stress increment, $\Delta \boldsymbol{S}_{A V E}$, and the macroscopic strain increment, $\Delta \boldsymbol{E}_{A V E}$, are calculated as average values of incremental stresses and incremental strains in each element as

$$
\Delta \boldsymbol{S}_{A V E}=(1 / N) \sum_{1}^{N}\left(\Delta \boldsymbol{S}_{i}\right) \text { and } \Delta \boldsymbol{E}_{A V E}=(1 / N) \sum_{1}^{N}\left(\Delta \boldsymbol{E}_{i}\right)
$$

where $N$ is the total number of Gaussian points.

Now, $\mu$ and $\beta$ are calculated using the incremental macroscopic plastic strain obtained from the following equation

$$
\left\{\Delta E_{A V E}^{P}\right\}=\left\{\Delta E_{A V E}\right\}-\left[D^{e}\right]^{-1}\left\{\Delta S_{A V E}\right\},
$$

where the macroscopic elastic matrix $\left[D^{e}\right]$ is assumed to be identical to that of the single crystal since only isotropic elastic materials are focused on in this study.

First, we have to determine the direction of the natural direction vector $\boldsymbol{N} n$ from the numerically obtained, macroscopic quantities $\Delta \boldsymbol{E}_{A V E}^{P}, \Delta \boldsymbol{S}_{A V E}$ and $\boldsymbol{S}_{A V E}$. Then, using $\Delta \boldsymbol{E}_{A V E}^{P}$ and $\Delta \boldsymbol{S}_{A V E}, \mu(\alpha)$ and $\beta(\alpha)$ are obtained as

$$
\begin{aligned}
& \mu(\alpha)=H_{M}^{\prime} /\left(\overline{\Delta S_{A V E}} / \overline{\Delta E_{A V E}^{P}}\right) \\
& \alpha=\cos ^{-1}\left\{(\sqrt{3 / 2})\left(\Delta \boldsymbol{S}_{A V E}^{\prime}: \boldsymbol{N} n\right) / \overline{\Delta S_{A V E}}\right\} \\
& \beta=\cos ^{-1}\left\{(\sqrt{2 / 3})\left(\Delta \boldsymbol{E}_{A V E}^{P}: \boldsymbol{N} n\right) /\left(\overline{\Delta E_{A V E}^{P}}\right\},\right.
\end{aligned}
$$

where $H_{M}^{\prime}$ is the macroscopic work-hardening coefficient obtained for loading in the natural direction, $\overline{\Delta S_{A V E}}=\sqrt{(3 / 2)\left(\Delta S_{A V E}^{\prime}\right)_{i j}\left(\Delta S_{A V E}^{\prime}\right)_{i j}}$ and $\overline{\Delta E_{A V E}^{P}}=$ $\sqrt{(2 / 3)\left(\Delta E_{A V E}^{P}\right)_{i j}\left(\Delta E_{A V E}^{P}\right)_{i j} \text {. }}$

\section{Numerical Results and Discussion}

Numerical calculations are performed in a twodimensional sub-space of Il'yushin's stress space as shown in Fig. 4. The definitions of initial loading direction angel $\theta_{s}\left(=\arctan \Sigma_{2} / \Sigma_{1}\right)$ and the natural direction angle $\theta_{n}\left(=\arctan N n_{2} / N n_{1}: N n_{i}\right.$ denotes the components of $\boldsymbol{N n})$ are also shown in Fig. 4, where $\Sigma_{1}$ $=S_{11}-\left(S_{22}+S_{33}\right) / 2$ and $\Sigma_{2}=\sqrt{3}\left(S_{22}-S_{33}\right) / 2$. The uniaxial stress, pure shear stress and three other stress states are chosen as fundamental stress states established by proportional loading.

Figure 5 shows stress-strain curves calculated for 


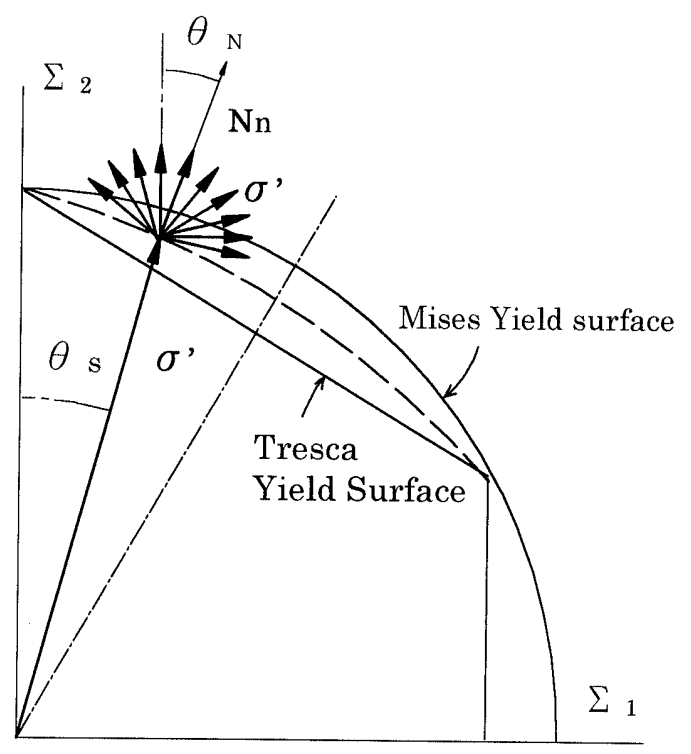

Fig. 4 Definition of initial loading direction angle $\theta_{S}$ and natural direction angle $\theta_{N}$ in a two dimensional subspace of Il'yshin's stress space

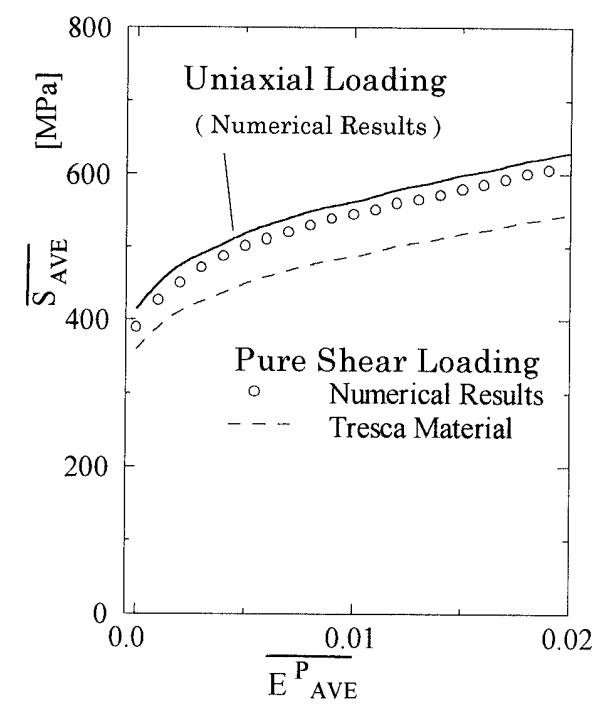

Fig. 5 Stress-strain curves for proportional loadings in a uniaxial stress case and in a pure shear stress case

uniaxial and pure shear loading. For comparison, the curve for pure shear loading of the Tresca material is also shown in the figure. The curves for the uniaxial loading and the pure shear loading cases locate fairly close with each other, and this suggests that a polycrystalline aggregate consisting of 64 single crystals is sufficient for the study of macroscopic constitutive properties and that the macroscopic iso-strain surface under proportional loading locates between the isotropic Mises-type and Tresca-type yield surfaces.

Figure 6(a) shows incremental stress-strain curves after the abrupt change of loading direction from a uniaxial one to a nonuniaxial one. The loading direction, namely the ratio of macroscopic stress increment $\Delta S_{22}$ to $\Delta S_{11}\left(\Delta S_{33}=0\right)$, is changed from $\alpha=$

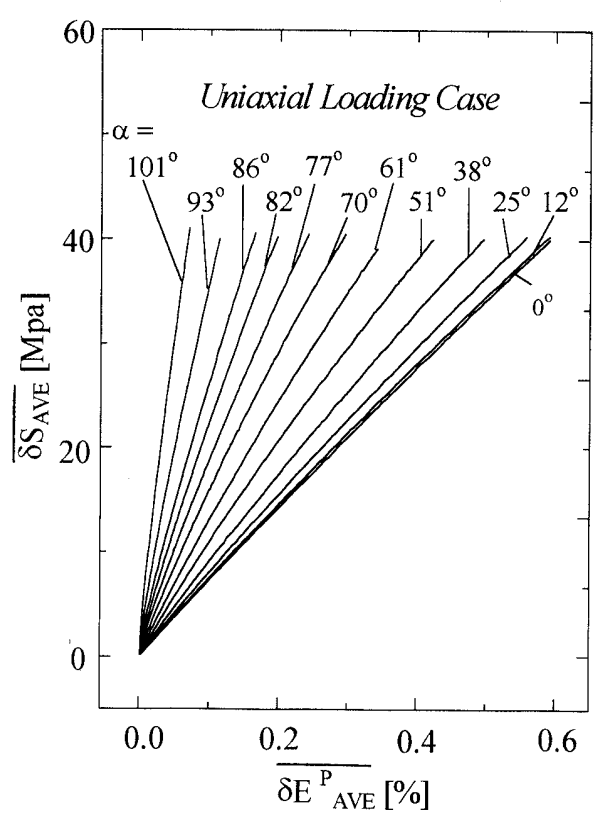

(a)

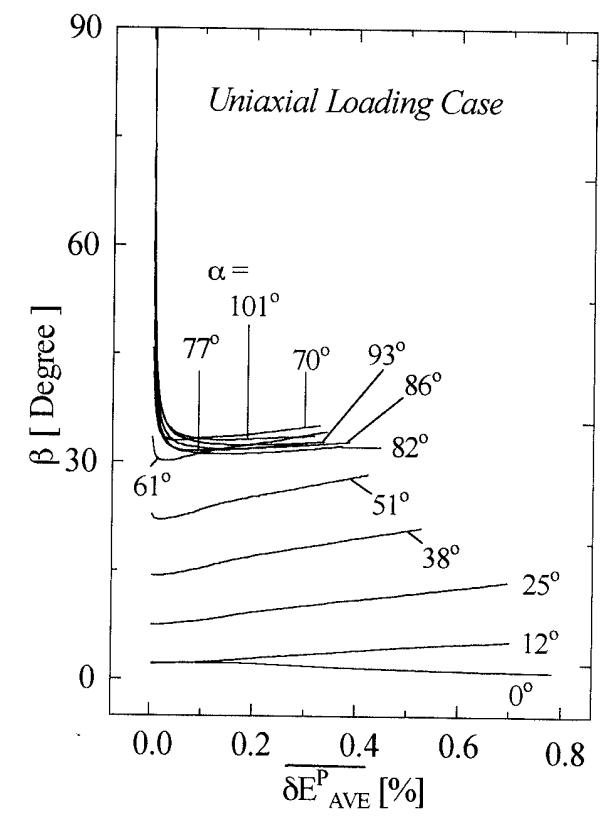

(b)

Fig. 6 Macroscopic incremental stress-strain relationships after abrupt change of loading direction from a uniaxial loading direction to a nonuniaxial one:

(a) Incremental stress-strain curves in terms of equivalent quantities (b) Variations of direction of incremental plastic strain for different stress increments

0 to $\alpha \neq 0$. The proportional loading curve is also plotted for comparison. As expected, the curves increases sharply at the point of direction change according to the degree of the change. Figure $6(\mathrm{~b})$ clearly shows that a change in $\alpha$ affects the plastic strain direction $\beta$. The results for the pure shear loading case are shown in Fig. 7. From a comparison 
of Fig. 6(b) and Fig. 7(b), it can be seen that a relatively strong directional dependence of the plastic strain increment is observed for the uniaxial loading case although the dependence is still observed for the pure shear loading case.

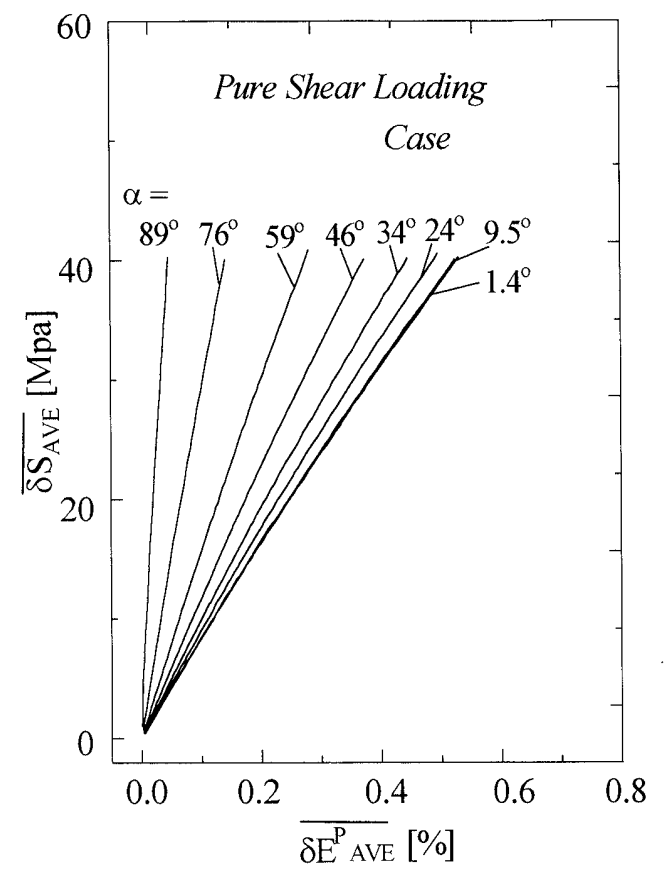

(a)

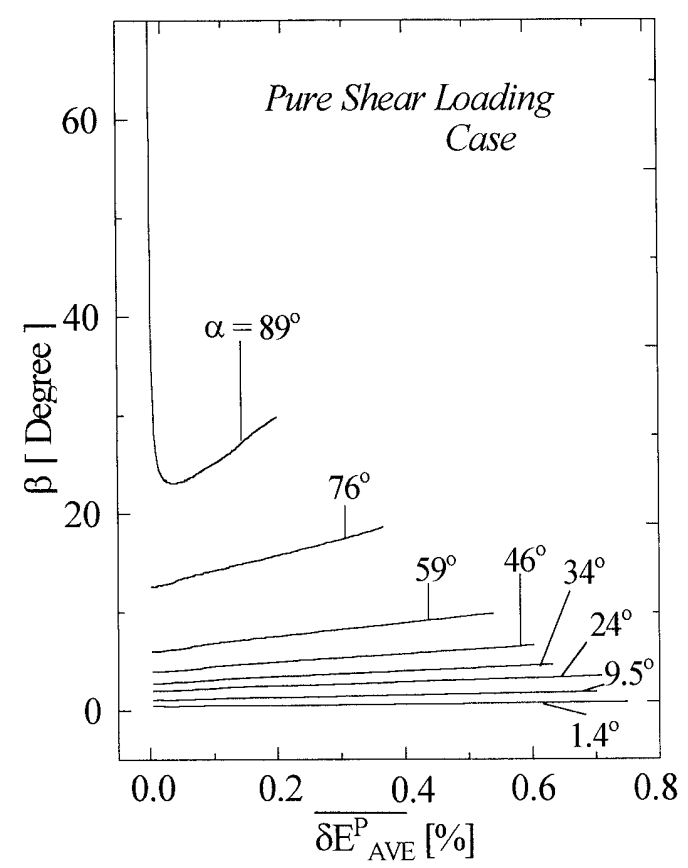

(b)

Fig. 7 Macroscopic incremental stress-strain relations after abrupt change of loading direction from a pure shear loading direction to a non-pure shear one :

(a) Incremental stress-strain curves in terms of equivalent quantities (b) Variations of direction of incremental plastic strain for different stress increments
Figure 8 shows the variations of $\mu(\alpha)$ and $\beta(\alpha)$ with respect to $\alpha$. The general tendency observed in the results appears to be the same as that reported in the past by Takahashi et al. ${ }^{(1)}$ using Lin's model and by Ito $^{(8)}$ using the KBW model. Figure 8(a) shows the results for two representative loading cases, namely the uniaxial loading and pure shear loading cases. It should be noted for the uniaxial loading case that small plastic strain increments appear for $\alpha \geq \pi / 2$; the yield loci may form a corner at the loading point in the stress space. It is interesting to note that the directional dependence is observed even for the pure shear loading case although the magnitude of the plastic strain for $\alpha=\pi / 2$ becomes almost zero, implying a locally smooth surface. Figures $8(\mathrm{~b}), 8(\mathrm{c})$ and 8 (d) show the numerical results for three loading cases between the uniaxial loading and the pure shear loading directions. The resulting curves show some intermediate tendency of those curves for the uniaxial loading and the pure shear loading cases. It is concluded from Fig. 8 that the variation of $|\beta|$ can be approximated by three straight lines in succession as a function of $|\alpha|$, namely $|\beta|$ linearly increases with the increase of $|\alpha|$ within the range $0^{\circ}<|\alpha|<60^{\circ}$, reaches $\left|\beta_{60}\right|$ when $|\alpha|=60^{\circ}$, then linearly increases again until $|\beta|$ reaches $30^{\circ}$ at $|\alpha|=90^{\circ}$ and remains at approximately $30^{\circ}$ for the range $90^{\circ}<|\alpha|$. Figure 9 shows the numerically obtained variation of $\beta_{60}$ with $\theta_{s}$ and it should be noted that $\beta_{60}\left(\theta_{S}\right)$ is a function of the initial loading direction angle $\theta_{s} . \quad \beta_{60}\left(\theta_{s}\right)$ decreases monotonically as the initial loading direction angle $\theta_{s}$ increases. In other words, the degree of directional dependence becomes weaker as the current stress vector locates closer to the pure shear stress state.

Furthermore, to complete the constitutive relation, we have to specify the relationships between the natural direction angle $\theta_{n}$ and the initial loading direction angle $\theta_{s}$. Figure 10 shows the numerically obtained relationships between $\theta_{n}$ and $\theta_{s}$. The numerical points locate between the theoretical lines predicted by the classical potential rules based on Tresca and Mises surfaces and $\theta_{n}$ increases as $\theta_{s}$ increases.

\section{Conclusions}

A finite-element polycrystalline model (FEPM) is developed to investigate the plastic constitutive properties such as directional dependence of the plastic strain increment on the stress increment direction, yield surface change after complex loading and Baushinger effects.

The numerical results obtained using the FEPM for face-centered cubic materials clearly show the directional dependence, namely $|\beta| \geq 0$ when $|\alpha|>0$ although the degree of the dependency may depend on 


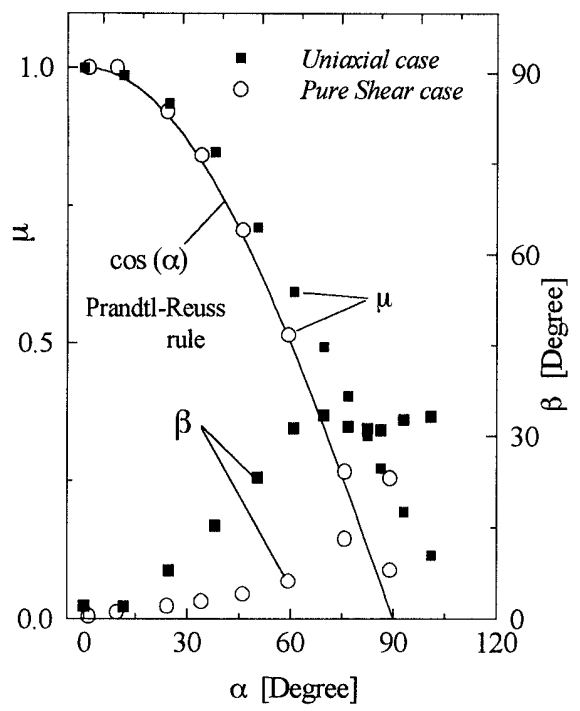

(a) $\theta s=0^{\circ}$ and $30^{\circ}$

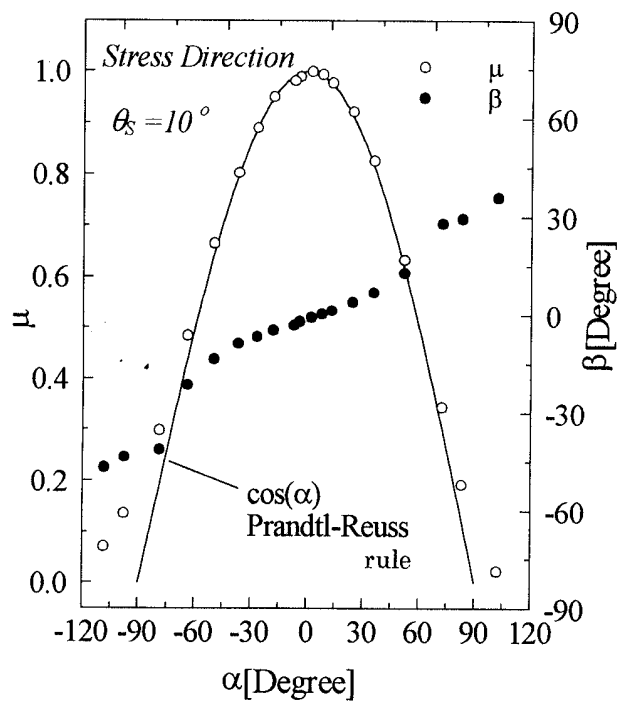

(c) $\theta s=10^{\circ}$

Fig. 8 Variations of the constitutive parameters $\mu(\alpha)$ and $\beta(\alpha)$

(a) for uniaxial loading and pure shear loading directions, $\theta_{s}=0$ and $\theta_{s}=30^{\circ}$, and for three other directions, (b ) $\theta_{s}=19^{\circ}$, (c) $\theta_{s}=10^{\circ}$ and (d) $\theta_{s}=50^{\circ}$

the fundamental loading direction. The results also show $\mu(\alpha) \geq \cos (\alpha)$ for any $\alpha$; for the same magnitude of stress increment, the plastic strain of the polycrystalline model can be larger than that predicted by the $J_{2}$-flow rule.

The numerical results for proportional loading cases suggest that, as a rough approximation for isotropic FCC materials, the Mises yield function may be utilized for judging whether a current stress satisfies the yield condition and accordingly that the natural direction may be replaced with the direction of the current stress and the directional dependence angle $\beta$ is determined based on the current stress

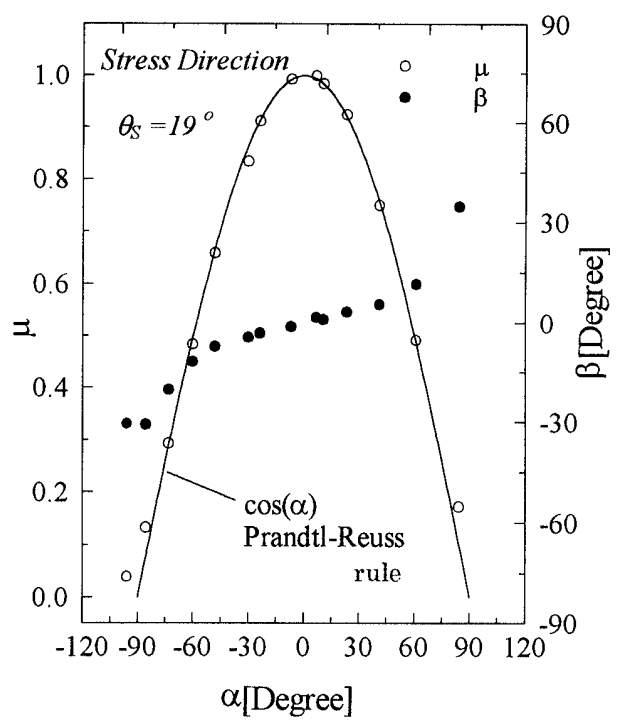

(b) $\theta s=19^{\circ}$

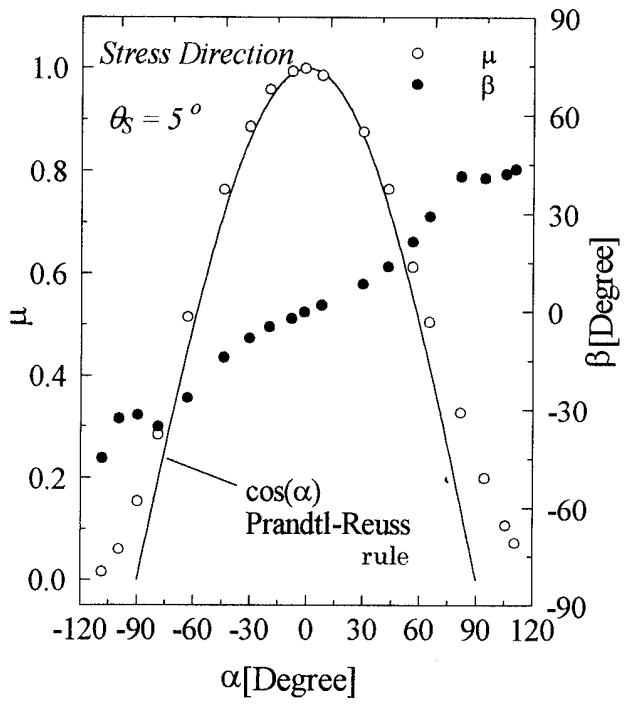

(d) $\theta s=5^{\circ}$

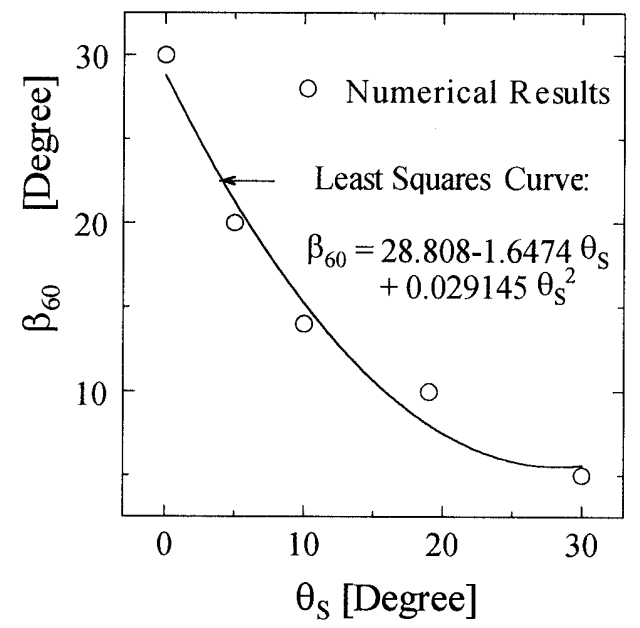

Fig. 9 Relationships between $\beta_{60}$, direction angle of plastic strain increment for stress increment with $\alpha=$ $60^{\circ}$ and initial loading direction $\theta_{S}$ 


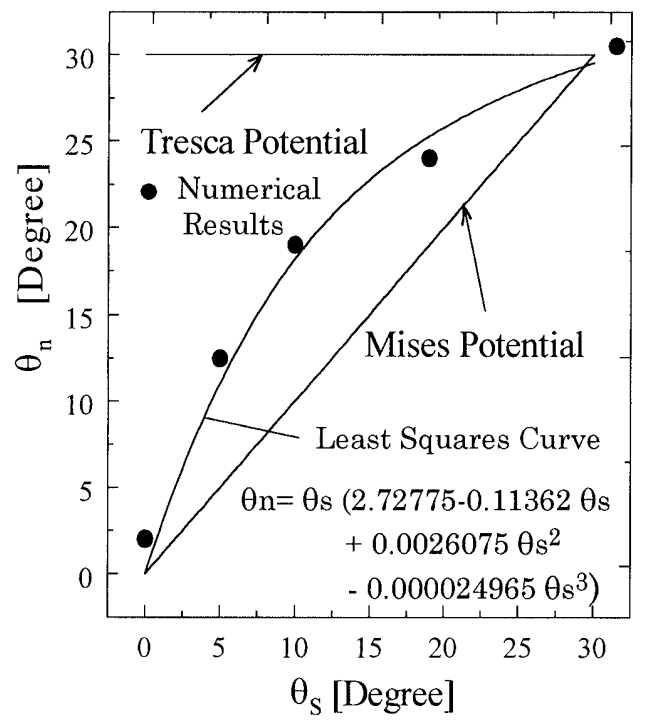

Fig. 10 Relationship between natural direction angle $\theta_{n}$ and initial loading direction $\theta_{s}$

direction. It should be noted, however, that if precise analysis formulas are required, the results in Figs. 9 and 10 for the yield condition and corresponding natural direction can be untilized. From the results shown in Fig. 8 , it is suggested that $\mu(\alpha)$ is simply approximated by $\cos (\alpha)$ for introduction of the directional dependence and the yield locus in the stress space is assumed to be smooth in any stress direction $\theta_{s}$. In relation to this approximation, for the range $90^{\circ}$ $\geq|\alpha|$, we may also approximate $\beta(\alpha)$ by simple connected lines by introducing the function $\beta_{60}\left(\theta_{s}\right)$ as shown in Fig. 9 and for the range $90^{\circ}<|\alpha|$ elastic unloading is assumed.

\section{References}

(1) Hill, R., On Discontinuous Plastic States, with Special Reference to Localized Necking in Thin Sheets, J. Mech. Phys. Solids, Vol. 1(1952), p. 19.

(2) Stören, S., and Rice, J.R., Localized Necking in Thin Sheets, J. Mech. Phys. Solids, Vol. 23(1975), p. 421.

(3) Batdorf, S.B. and Budiansky, B., A Mathematical Theory of Plascticity Based on the Concept of Slip, NASA TN 1871(1949).

(4) Christoffersen, J. and Hutchinson, J.W., A Class of Phenomenological Corner Theories of Plasticity, J. Mech. Phys. Solids, Vol. 27 (1979), p. 465.

(5) Gotoh, M., A Class of Plastic Constitutive Equations with Vertex Effect, Int. J. Solids Structures, Vol. 21(1985), p. 1101.

(6) Gotoh, M., Some Improvements of J 2 F/J 2 D for FEM Analyses of Large Elastic-Plastic Deformation Including Strain Localization, Proc. Plasticity 89, Eds. Khan, A.S. and Tokkuda, M., (1989), p. 443.
(7) Goya, M. and Ito, K., An Expression of Constitutive Law of Elastic-Plastic Material-Initially Isotropic Materials with Mises Type Plastic Potential, JSME, Int. J., Ser. I(1990), p. 57.

(8) Ito, K., New Flow Rule for an Elastic-Plastic Solid Based on KBW Model with a View to Lowering the Buckling Stresses of Plates or Shells, The Technology Reports of the Tohoku University, Vol. 44 (1979), p. 199.

(9) Kroner, E., Zur Plastishen Verfomung des Vielkristalls, Acta Metall., Vol. 9(1961), p. 155.

(10) Budiansky, B. and Wu, T.T., Theoretical Prediction of Plastic strains of Polycrystals, Proc. 4th U. S. Nat. Congr. Appl. Mech. Vol. 2(1962), p. 1175.

(11) Takahashi, H., Ito, K. and Goya, M., Directional Dependence of Plastic Strain-Rate Vector on Stress-Rate Vector: Numerical Experiments based on Lin's Polycrystal Model, Int. Jour. Plasticity, Vol. 6(1990), p. 615.

(12) Takahashi, H., Saitoh, T., Motohashi, H., Tokuda, M. and Abe, T., Prediction of Plastic Anisotropy in an Aluminium Sheet by Finite Element Polycrystal Model, Jour. JSME(in Japanese), Vol. 60 (1994), p. 1017.

(13) Havner, K.S., A Discrete Model for the Prediction of Subsequent Yield Surfaces in Polycrystalline Plasticity, Int. J. Solids Structures, Vol. 7(1971), p. 719 .

(14) Jimma, T., Murota, T. and Ichiyanagi, T., StressStrain Stiffness Matrix of Metal Crystals and its Applications, J. Jap. Soc. Mech. Engrs. (in Japanese), Vol. 75(1972), p. 602.

(15) Miyamoto, H., Ihijima, Y. and Miyoshi, T., Mechanical Properties of Materials Studied from the Viewpoint of their Constituents (Three Types of Models), J. Jap. Soc. Mech. Engrs. (in Japanese), Vol. 75 (1972), p. 575.

(16) Gotoh, M., A Finite Element Formulation for Large Elastic-Plastic Deformation Analysis of Polycrystals and Some Numerical Considerations on Polycrystalline Plasticity, Int. Jour. Num. Meth. Eng., Vol. 12(1978), p. 101.

(17) Asaro, R.J., Geometrical Effects in the In homogeneous Deformation of Ductile Single Crystals, Acta Metall., Vol. 27 (1979), p. 445.

(18) Asaro, R.J. and Rice, J.R., Strain Localization in Ductile Single Crystals, J. Mech. Phys. Solids, Vol. 25 (1977), p. 309.

(19) Balasubramanian, S. and Anand, L., Single Crystal and Polycrystal Elasto-Viscoplasticity, Application to Earing in Cup Drawing of F.C.C. Materials, Comp. Mechanics, Vol.17(1996), p. 209.

(20) Yamada, Y., Yoshimura, N. and Sakurai, T., Plastic Stress-Strain Matrix and its Application for the Solution of Elastic-Plastic Problems by the Finite Element Method, Int. J. Mech. Sci., Vol. 10 (1968), p. 343. 